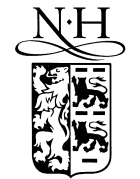

ELSEVIER

\title{
Correction of wavefront distortions by means of thermally adaptive optics
}

\author{
H. Lück ${ }^{\text {a,b }}$, K.-O. Müller a,c , P. Aufmuth ${ }^{a, *}$, K. Danzmann ${ }^{\text {a,b }}$ \\ ${ }^{\text {a }}$ Universität Hannover, Institut für Atom- und Molekülphysik, Abt. Spektroskopie, Callinstr. 38, D-30167 Hannover, Germany \\ ${ }^{\mathrm{b}}$ Max-Planck-Institut für Quantenoptik, Aussenstelle Hannover, Callinstr. 38, D-30167 Hannover, Germany \\ ${ }^{\mathrm{c}}$ Technische Universität Darmstadt, Institut für Angewandte Physik, Abt. Licht- und Teilchenoptik, Hochschulstr. 6, \\ D-64289 Darmstadt, Germany
}

Received 27 October 1999; received in revised form 30 December 1999; accepted 3 January 2000

\begin{abstract}
A new method for the correction of wavefront distortions in laser interferometers is proposed. In contrast to the usual adaptive correction, by means of piezoelectric actuators, a high power laser beam is used to control the thermal expansion of the optical elements. Since the correction is performed without mechanically contacting the element, no additional mechanical noise is introduced. Initial measurements on aluminium-coated mirrors show the feasibility of this 'thermally adaptive optics' method. (C) 2000 Elsevier Science B.V. All rights reserved.
\end{abstract}

PACS: 04.80.Nn; 07.60.Ly; 42.79.Ls; 44.50.+ f

Keywords: Michelson interferometer; Wavefront distortions; Adaptive optics; Thermal lensing; Gravitational wave detector

\section{Introduction}

The overall performance of optical instruments is often limited by the quality of the reflecting surfaces and by the non-homogeneity of the transmitted optical elements. This leads to wavefront deformations of a propagating light field. If these imperfections are static they can be corrected by means of an appropriate mask. However, often they change with time. In these cases the corrections have to be adapted continuously according to the varying optical conditions.

\footnotetext{
* Corresponding author. Tel.: +49-511-762-2386; fax: +49511-762-2784; e-mail: pea@mpq.mpg.de
}

A severe problem in high power laser systems is absorption in the optical components leading to an unpredictable local heating and hence to deformations. These cause a wavefront distortion of the reflected or transmitted beam. In addition, since the optical substrates have a finite thermal conductivity, there will be a temperature gradient inside the substrate. The corresponding gradient of the refraction index has an effect like a lens. This leads to a change in the wavefront curvature of the transmitted beam, so-called thermal lensing.

In optical astronomy several techniques ('adaptive optics') have been developed for the correction of static or time-dependent optical aberrations; e.g. for the large telescopes one has to compensate for the fluctuations of the refractive index of the atmosphere 
[1]. Another field of application of adaptive techniques is the enhancement of Michelson interferometry [2]. In most of these cases piezoelectric actuators press onto the back of the mirror and hence deform the surface in order to outbalance the changing imperfections.

In some applications, however, the direct action of the actuators onto the mirrors produces intolerable mechanical disturbances. This is the case in laser-interferometric gravitational wave detectors where the use of actuators introduces too much noise. Therefore, we propose a new means to correct the surface deformations without mechanically contacting the mirror, the so-called 'thermally adaptive optics' method [3]. It uses an intense laser beam that causes thermoelastic deformations of the mirror surface to correct the measured aberrations. First experiments show the feasibility of this method.

\section{Thermal effects in laser interferometers}

Since the mid-nineties large laser interferometers are built in order to detect gravitational waves, predicted by Albert Einstein in his general theory of relativity (see, e.g. [4]). These waves are created by accelerated masses producing ripples in the fabric of spacetime itself. They periodically change the distance $l$ between test bodies, stretching it in one direction and squeezing it in the perpendicular one. Thus a Michelson interferometer is an ideal instrument for detecting these changes as an arm length difference $\Delta l$, where mirrors serve as test masses. Since the effect is extremely small (typically $\Delta l / l \sim$ $10^{-21}$ ) arm lengths of some kilometers are necessary. The operation of interferometric gravitational wave detectors requires an exceptionally highpowered light source because the sensitivity of the instrument depends on the amount of light energy stored in the arms [5]. The optimal power that minimises photon shot noise and radiation pressure noise simultaneously (the so-called 'standard quantum limit') may be as high as several megawatts [6].

Up to now the continuous wave lasers used in the currently built detectors produce only about $10 \mathrm{~W}$ light power, but with the power recycling technique it is possible to increase the circulating power up to $10 \mathrm{~kW}$ or even higher [7]. Power recycling makes use of the fact that the output of the interferometer is kept on a dark fringe. Thus all the light is reflected back towards the laser and can be used again. An additional mirror in front of the input port and the interferometer itself form an optical cavity that provides a resonant enhancement of the laser light.

The light power thus attainable is limited by surface deformations and thermal effects. Even when the absorption of the transmitted optical elements, like the beam splitter and compensation plate, is as low as technically achievable $(\sim 1 \mathrm{ppm} / \mathrm{cm})$, the formation of thermal lenses [8] is unavoidable with such high power levels, and the absorption in the coating leads to local deformations of the mirror surfaces. The wavefront distortions thus induced reduce the interference contrast at the output, increase the losses in the power recycling cavity and reduce the sensitivity of the interferometer.

Thermal lensing cannot be compensated for in advance by appropriately shaping the optical elements. This would lead to poor interference contrast for low light power and hence to a reduced power build-up. Such a pre-shaping will render the cold cavity optically unstable which makes the start-up process very difficult. The use of adaptive optics could improve the situation two-fold: First, in the case of poor interference contrast due to poor quality of the end mirrors, one of the mirror surfaces has to be deformed in order to match the distortions of the other one. This can be achieved by applying a static deformation. Second, the thermal lenses can be compensated for either by deforming the reflecting mirrors or by heating the elements themselves in order to balance the thermal input. As the absorption changes in time with the power build-up in the cavity, a dynamic compensation is required.

Unfortunately, in interferometric gravitational wave detectors the high mechanical $Q$ values of the optical components, required to keep the thermal noise sufficiently low, prohibit the use of standard adaptive equipment like piezoelectric elements. These would introduce too much mechanical noise. As a non-contact method of controlling the mirror surface we propose to use thermally induced deformations in a constructive way, i.e. to produce controlled expansion of the mirror by means of a laser beam. Varying the power or the exposure time at a given position then allows the shaping of the surface according to the requirements. 


\section{Theory of thermoelastic deformations}

In this section the basic equations for the calculation of thermal deformations of cylindrical solids are presented. As an example the heating of a massive mirror by means of an intense laser beam is discussed. In this case the laser power is partly absorbed by the bulk material and partly by the coating.

A very simple but effective estimate of the thermal expansion $\delta s$ of the curvature depth (sagitta) of a spherical mirror under the influence of a laser beam has been given by Winkler et al. [9]:

$\delta s=\frac{\alpha}{4 \pi K} P_{\text {abs }}$

where $\alpha$ is the thermal expansion coefficient, $K$ the thermal conductivity, and $P_{\mathrm{abs}}$ the absorbed power. According to this formula, for a fused silica mirror in a confocal set-up an absorbed power of $3 \mathrm{~W}$ leads to a local deformation within the beam diameter that compensates the original curvature; the mirror can then be considered as being locally flat. A similar wavefront distortion $\delta s$ will be caused by a thermal lens due to bulk absorption of about $0.1 \mathrm{~W}$.

In general, for the calculation of temperature distributions the Lamé-Navier equations have to be solved:

$\mu \Delta \boldsymbol{u}+(\lambda+\mu) \nabla(\nabla \cdot \boldsymbol{u})+\gamma \nabla T+\rho \boldsymbol{f}=0$

where $\lambda$ and $\mu$ are the first and the second Lamé coefficient, respectively; $\gamma=(3 \lambda+2 \mu) \alpha ; \boldsymbol{u}$ the displacement vector; $T$ the temperature; $\rho$ the density; and $f$ the external forces. The solutions given in the literature differ considerably according to the boundary conditions and the simplifications made by the different authors.

Hello and Vinet [10] give analytical expressions for the induced thermal lenses and thermoelastic deformations of a coated cylindrical mirror heated by an axially symmetrical cw Gaussian laser beam, both for the steady state and for the transient regime. The mirror is supposed to be suspended in a vacuum tank as part of a high power gravitational wave detector. Thus the model only takes care of radiation losses, but it is also valid for convection losses. The boundary conditions follow from the assumption that there are no external forces $(f=0)$.
The main result of Hello and Vinet's study is that the thermoelastic deformations caused by bulk absorption of laser light in pure silica are about 5 times weaker than those caused by dissipation in the coating, for the same absorbed power. The resulting wavefront aberrations are at least a factor of 10 weaker than those caused by the thermal lens effect [10]. Thus thermal lensing represents the main cause of wavefront distortions in high power laser interferometers.

\section{Aims and principle}

Our aim is the improvement of the interference pattern at the output port of an interferometric gravitational wave detector. This means to enhance the interference contrast and to correct thermal lensing.

Usually, the visibility $\mathscr{V}$ is taken as a measure for the quality of the interference contrast

$\mathscr{V}=\frac{I_{\max }-I_{\text {min }}}{I_{\max }+I_{\min }}$

where $I_{\max }$ is the constructive-interference intensity and $I_{\min }$ that of destructive interference, assuming equal reflectivity for both mirrors.

In modern high-finesse interferometers, like the Garching $30 \mathrm{~m}$ prototype gravitational wave detector [11], a visibility of virtually 1 has been obtained. This means that even small mirror deformations $(\delta s \sim \lambda / 100)$ lead to a marked reduction of $\mathscr{V}$. For advanced interferometric gravitational wave detectors operating at the limits of the technically achievable sensitivity this means a substantial decrease in the signal to noise ratio.

The essential aim is the correction of thermal lensing. Large changes $\delta s$ in the effective mirror curvature will bring the recycling cavity close to instability. From (2), the maximum single-pass thermal lensing that can be tolerated for fused silica corresponds to an absorbed power of about $90 \mathrm{~mW}$ [8]. For a circulating power of $10 \mathrm{~kW}$, absorption losses of $1 \mathrm{ppm} / \mathrm{cm}$ and a substrate thickness of $10 \mathrm{~cm}$, this limit will be exceeded.

The correction of thermal lensing can be done in several ways: Firstly, the optical component can be suitably pre-shaped in order to anticipate the effect; this requires conversely an additional start-up heat- 
ing of the element as long as the circulating power is low. Secondly, the compensation can be performed on the lensing element itself by means of adaptive optics. Thirdly, the distortion introduced by one element can be compensated for by the adaptation of other optical elements.

We have developed an adaptive optical system based on selective thermal deformation. A Michelson interferometer serves as a measuring unit to detect the wavefront distortions of one of the mirrors, as described in Section 5. For each point of the mirror surface a computer program calculates from these data the necessary corrections and converts this information into control signals for the adjusting unit. The latter consists of a high power laser and a two-dimensional scanner that allows the heating of each point of the surface selectively. By means of a control loop the deviations can be suppressed continually.

\section{Wavefront detection method}

For our purposes we have to know the sign of the wavefront distortions as well as their amplitude. The following method gives both quantities at the same time. It uses the principle of internal modulation at low frequencies, but also works with very high frequencies, e.g., with Schnupp modulation [7].

Consider a Michelson interferometer with arm lengths $L$ and $L^{\prime}$, illuminated by a laser with intensity $I_{0}$ and wave length $\lambda$. At the output port the intensity is given by

$I_{\text {out }}=\frac{I_{0}}{2}(1-\cos (\Delta \phi))$

where $\Delta \phi=2 k \Delta L$ is the phase difference introduced in the arms, with $k=2 \pi / \lambda$ and $\Delta L=L-L^{\prime}$. From (4) one obtains

$\Delta \phi=\arccos \left(-\frac{2 I_{\text {out }}}{I_{0}}+1\right) \quad(0 \leq \phi \leq \pi)$.

A control loop stabilises the output port to a dark fringe corresponding to a phase $\phi_{0}=0$ (i.e. $L=L^{\prime}$ without loss of generality). The position of one of the end mirrors is now modulated periodically with an amplitude $A=a \lambda /(4 \pi)$, i.e. the phase shift introduced by the modulation is $a$. A CCD camera takes a picture of the intensity distribution $I$ at the interferometer output.

We measure this intensity distribution at the two turning points of the mirror moving back and forth, at the front position:

$I_{1}(\Delta \phi)=\frac{I_{0}}{2}(1-\cos (\Delta \phi+a))$

and at the back position:

$I_{2}(\Delta \phi)=\frac{I_{0}}{2}(1-\cos (\Delta \phi-a))$.

$\Delta \phi \equiv \Delta \phi(x, y)$ is now the deviation of the phase from $\phi_{0}$, i.e. $\Delta \phi+a=2 k \Delta L_{1}$ and $\Delta \phi-a=$ $2 k \Delta L_{2}$. The difference between $I_{1}$ and $I_{2}$ is

$\Delta I(\Delta \phi)=I_{0} \sin (\Delta \phi) \sin (a)$.

The reverse function gives the desired value of the phase deviation as well as its sign

$\Delta \phi(\Delta a I)=\arcsin \left(\frac{\Delta I}{I_{0} \sin a}\right)\left(-\frac{\pi}{2} \leq \Delta \phi \leq \frac{\pi}{2}\right)$.

The signal to noise ratio depends on the phase modulation amplitude $a$. The optimal value of $a$ results from the magnitude of the deviations. Without loss of generality the largest deviation may have a positive value $\phi_{\max }$. Then the maximum intensity is $I_{\max }=\left(I_{0} / 2\right)\left(1-\cos \left(\phi_{\max }+a\right)\right)$. If for each pixel of the image $n$ grades of brightness are available $(0$ to $n-1)$ and if the exposure time of the camera is such that the pixels have the brightness value $n-1$ for $I_{\max }$ then the minimum intensity noise $N=$ $I_{\max } / n$. With the signal $S$ from (8) one obtains

$\frac{S}{N}=2 n \sin (\Delta \phi) \frac{\sin \alpha}{1-\cos \left(\phi_{\max }+a\right)}$.

Hence the optimal modulation amplitude $A_{\text {opt }}=$ $\phi_{\max } \lambda /(4 \pi)$ and

$\left(\frac{S}{N}\right)_{\mathrm{opt}}=n \frac{\sin (\Delta \phi)}{\sin \left(\phi_{\max }\right)}$. 
A useful approximation of (9) for small deviations from $\phi_{0}$ is

$$
\Delta \phi \cong \frac{\Delta I}{I_{0} \sin a} .
$$

With (12), the time for the calculation of the control signal for the adjusting unit is drastically reduced since $a$ and hence $\sin a$ are constant. Especially for small wavefront distortions this approximation is sufficiently good.

An electronic control loop (Section 6.1) stabilises the phase front to a mean value

$\bar{\phi}=\frac{1}{m} \sum_{i=1}^{m} \phi_{i}=0$.

The $\phi_{i}$ are the phase deviations of the $m$ points the camera can resolve. A criterion for the quality of the adaption is the standard deviation

$\sigma=\left(\frac{1}{m} \sum_{i}\left(\phi_{i}-\bar{\phi}\right)^{2}\right)^{1 / 2}$.

For small deviations $\Delta \phi$ the output intensity is $\Delta I(\Delta \phi) \cong \frac{1}{4} I_{0} \sigma^{2}$. Thus with $\sigma$ one can determine the residual brightness and hence the visibility $\mathscr{V}$.

With this method the correction can be done only in one direction. According to the wavefront detection scheme the maximum phase difference that can be corrected is $\pi$ (cf. (9)) corresponding to a mirror expansion of $\lambda / 4$. The maximum lift can only be applied to a spot with $\Delta \phi=-\pi / 2$.

\section{Experimental arrangement}

Fig. 1 shows the experimental arrangement. In the following the components of the apparatus are described in detail.

\subsection{Michelson interferometer}

The main component is a table-top Michelson interferometer with aluminium-coated mirrors of $2.54 \mathrm{~cm}$ diameter. The expanded beam from a $\mathrm{He}-\mathrm{Ne}$ laser $(\lambda=632.8 \mathrm{~nm})$ serves as a light source covering the whole area of the mirrors, thus creating an interference pattern of equal size at the output port. The interferometer is operated in vacuum in order to avoid convection losses and acoustic disturbances.

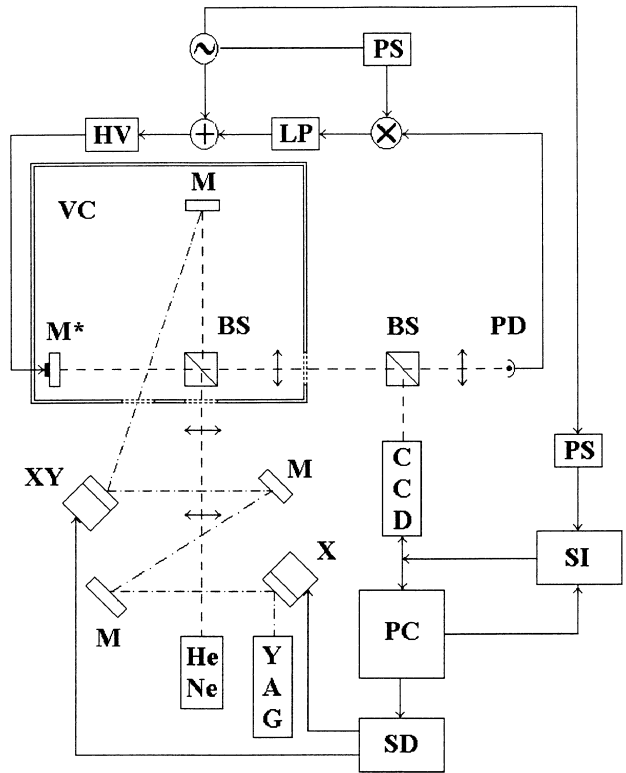

Fig. 1. Experimental arrangement. BS, beam splitter; CCD, camera; HeNe, He-Ne laser; HV, high voltage amplifier; LP, low pass filter; $M$, mirror; $M^{*}$, mirror with piezoelectric transducer; YAG, Nd:YAG laser; PC, personal computer; PD, photo diode; PS, phase shifter; SD, scanner drive; SI, signal inverter; VC, vacuum chamber; $\mathrm{X}, x$-scanner; $\mathrm{XY}, x y$-scanner. The vacuum chamber VC houses a Michelson interferometer (BS-M-M*) illuminated by the $\mathrm{He}-\mathrm{Ne}$ laser. The piezo element at $\mathrm{M}^{*}$ modulating the mirror position serves to obtain an error signal. The upper circuit represents the control loop. The output of the Michelson (to the right) is split up (BS) and recorded by a photodiode (PD) and a CCD camera. The signal depends on the wavefront distortions produced at the mirror $\mathrm{M}$. The PC calculates how and where to deform $\mathrm{M}$ in order to outbalance these distortions. The deformation is done by heating $\mathrm{M}$ with the Nd:YAG laser via the scanners $\mathrm{X}$ and $\mathrm{XY}$, controlled by the PC with the scanner drive SD.

The arm lengths are about $18 \mathrm{~cm}$; by means of white light interferometry equal arm lengths are achieved. This helps to make the interferometer insensitive to fluctuations of the longitudinal modes of the $\mathrm{He}-\mathrm{Ne}$ laser. The interferometer is operated at a dark fringe, i.e. with destructive interference at the detection port. An electronic control loop stabilises the interferometer to this operating point (this corresponds to $\bar{\phi}=0$ ). The error signal is obtained with a $400 \mathrm{~Hz}$ modulation of the mirror position by means of a piezoelectric actuator. (For the application in a gravitational wave detector this would be replaced by a modulation method involving no mechanical contact.) 


\subsection{Wavefront sensor}

The above-mentioned modulation of the mirror position is also used for the wavefront detection. A gated intensified CCD camera and an image processing card are triggered at both turning points of the moving mirror. The minimum gate time of the camera is $5 \mathrm{~ns}$. The image converter card has a dynamic range of 8 bits. The exposure time for constructive interference was chosen so as to make optimal use of this range. This time is about $9 \mu$ s and thus so short that there are no changes in the brightness of the pixels caused by the motion of the mirror.

In order to obtain an optimal signal-to-noise ratio the modulation amplitude $a$ was set to a value near $\pi / 2$, i.e. the lateral lift $A=a /(2 k)=\lambda / 8$. A lens half way between the mirror and the camera images the interference pattern at the surface of the mirror 1:1 onto the camera. The aperture of the camera limits the pattern to a circle of $1 \mathrm{~cm}$ diameter.

The images of the interference pattern at both turning points of the mirror are recorded and then subtracted, for each pixel according to the formula $\Delta I=128+\frac{1}{2}\left(I_{2}-I_{1}\right)$. This reduction of the dynamic range by a factor of 2 can be tolerated because of the rather high noise level of the camera. Since the subtraction of the signals and the recording of the difference image are done directly on the image processing card these processes run at high speed. The phase of the wavefront can be computed with (9) or for small deviations with (12) and then be used to deduce a control signal.

\subsection{Control and deflection unit}

The thermal adjustment of the mirror surface is done by means of a cw Nd:YAG laser. For the first experiments we used a monolithic non-planar miniature ring laser [12] because of its high stability with regard to amplitude and mode behaviour. It turns out that a rather low output power (about $1 \mathrm{~W}$ ) is sufficient to perform the desired thermal deformations, because the $1064 \mathrm{~nm}$ radiation is appreciably absorbed by the aluminium-coated mirror. In the

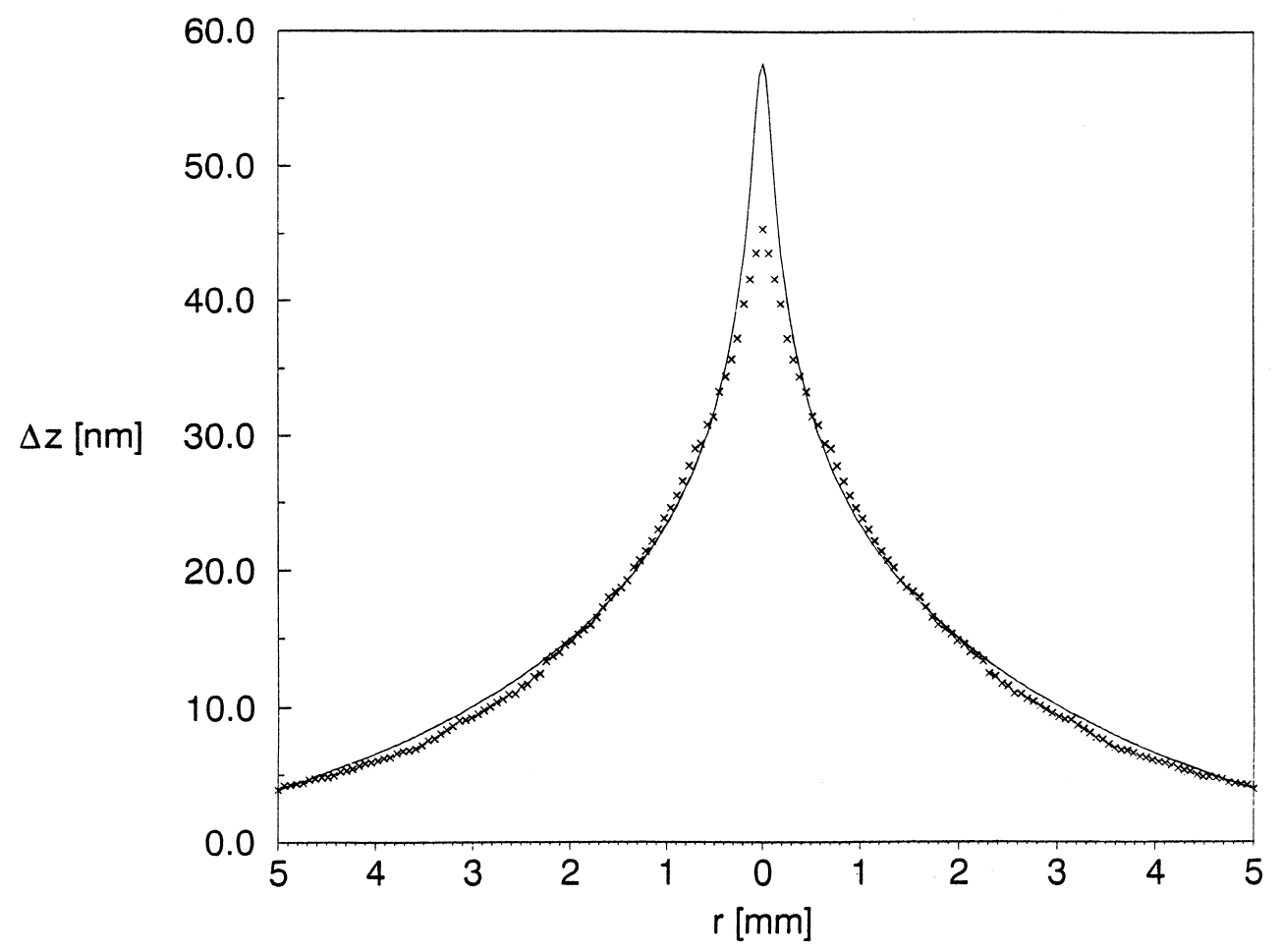

Fig. 2. Deformation of the central part of a BK7 mirror coated with aluminium by means of $100 \mathrm{~mW}$ Nd:YAG laser power. Comparison of theory [10] (plain line) and experiment (crosses); $r$, mirror radius; $\Delta z$, mirror expansion. 
planned gravitational wave detectors a Nd:YAG laser serves as the light source [13] because low-loss dielectric coatings will be used; then a different heating laser is required (for example a $\mathrm{CO}$ or $\mathrm{CO}_{2}$ laser).

The laser beam is positioned by means of an $x y$ galvo-drive (G120DT, General Scanning) controlled by a self-written computer program. The amount of deformation depends on the laser power and the exposure time. For the case that no heating is required the laser beam can be deflected into a beam dump instead to the $x y$-scanner ('blanking') by means of an additional $x$ galvo-drive (G108, General Scanning), since even the smallest possible irradiation already causes a deformation. Both scanner mirrors are dielectrically coated for $\lambda=1064 \mathrm{~nm}$ and high laser power. Both have only a small aperture $(5 \mathrm{~mm})$ in order to obtain a high scan velocity.

For the scanning of the mirror surface one has two possibilities, either the definition of a rigid grid or of a fixed number of points without specific positions. A grid has the advantage that only the exposure time has to be computed. Repeated measurement of the wavefront leads to continuously corrected values for this time. With the second method one can selectively aim at those points that have to be deformed, but this requires much more computing time. After each phase measurement the coordinates as well as the irradiation times have to be determined. In either case the optimal number of points has to be found experimentally. A larger number leads to a more precise correction but also to a lower repetition rate. This entails cooling of the irradiated spots between consecutive scans.

\section{Experiments and results}

We present first experiments using thermally adaptive optics showing the feasibility of this method.

\subsection{Sample and mounting}

We chose a BK7 substrate because of its high thermal coefficients $\left(\alpha=7.10 \times 10^{-6} \mathrm{~K}^{-1}\right.$ and $K$ $=1.114 \mathrm{~W} / \mathrm{mK}$ ). The mirror with $25.4 \mathrm{~mm}$ diameter and $6.5 \mathrm{~mm}$ thickness was coated with aluminium. The Nd:YAG laser beam was focussed onto the mirror surface. At $1064 \mathrm{~nm}$ the coating absorbs

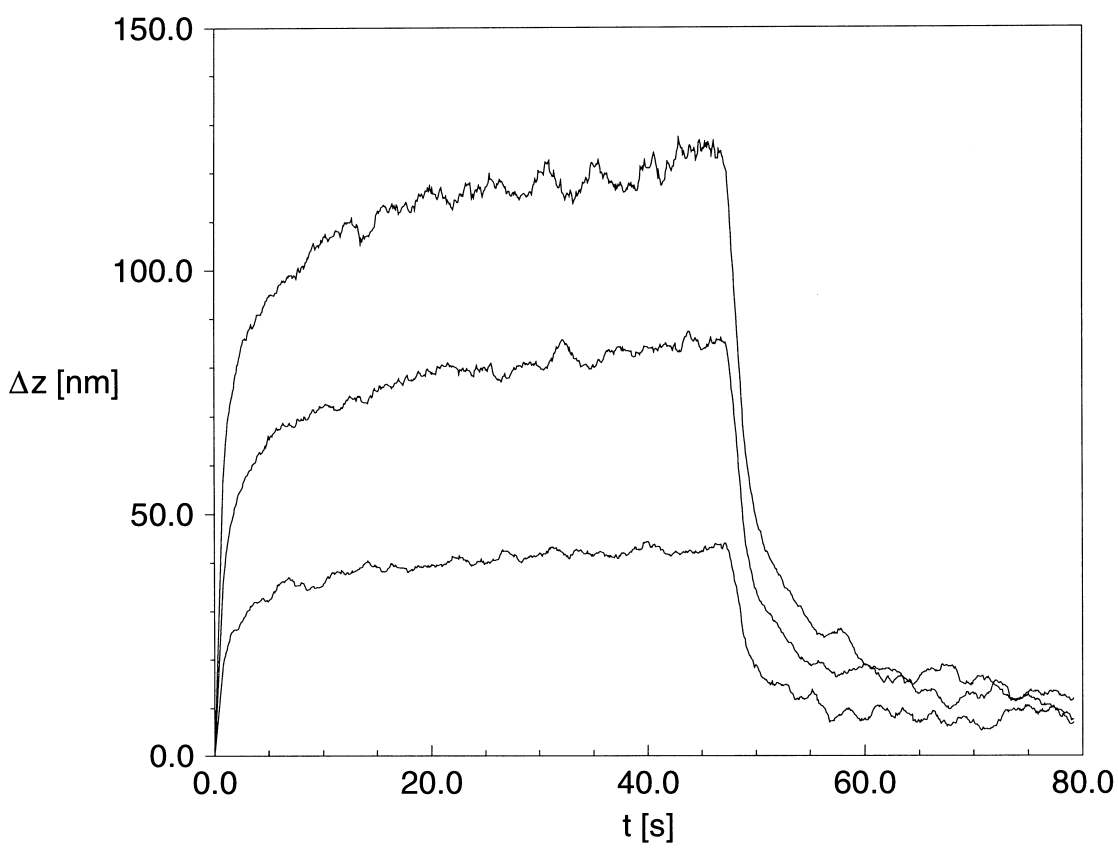

Fig. 3. Transient behaviour of the maximum thermal deformation of a BK7 mirror coated with aluminium. Exposure time: 48 s; cooling time: $32 \mathrm{~s}$; laser power: $100 \mathrm{~mW}, 200 \mathrm{~mW}, 300 \mathrm{~mW}$ (from bottom to top); $t$, time; $\Delta z$, mirror expansion. 
$10 \%$ of the laser power and converts it to heat. The measurements have been performed at a pressure of about 1 mbar.

The mounting should have as little contact with the mirror as possible allowing the heat to be radiated freely. Otherwise the points of contact produce a heat sink leading to an uncontrolled deformation. Furthermore, the holder should not exert forces on the mirror producing internal strain. The optimal solution is a monolithic suspension with fused silica fibres as it is planned for GEO 600 [14]. Since this requires an additional longitudinal control loop we did the experiment without such a suspension. The mirror fixture consists of a ring with four thin rods holding and fixing the mirror. The disturbance introduced by these supporting points is negligible. The

(a)
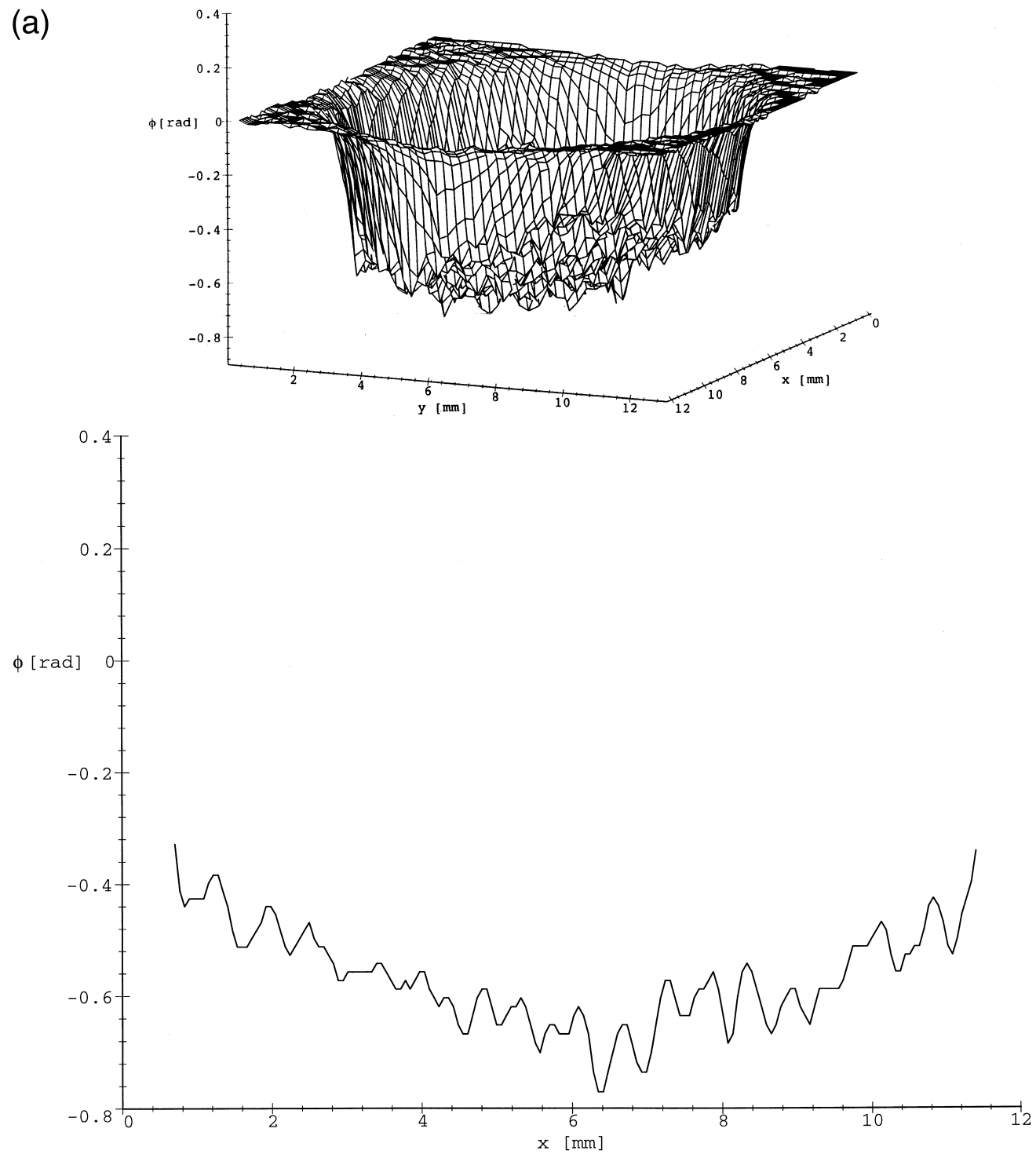

Fig. 4. (a) Phase front of a displaced mirror; the mean value of the deformations is $\bar{\phi}=-0.588$ rad and the standard deviation $\sigma$ of the wavefront from $\phi=0 \mathrm{rad}$ is $0.556 \mathrm{rad} ; x, y$, mirror co-ordinates; $\phi$, phase. (b) Corrected phase front of the displaced mirror (a); $\bar{\phi}=-0.026 \mathrm{rad}$ and $\sigma=0.055 \mathrm{rad}$. 

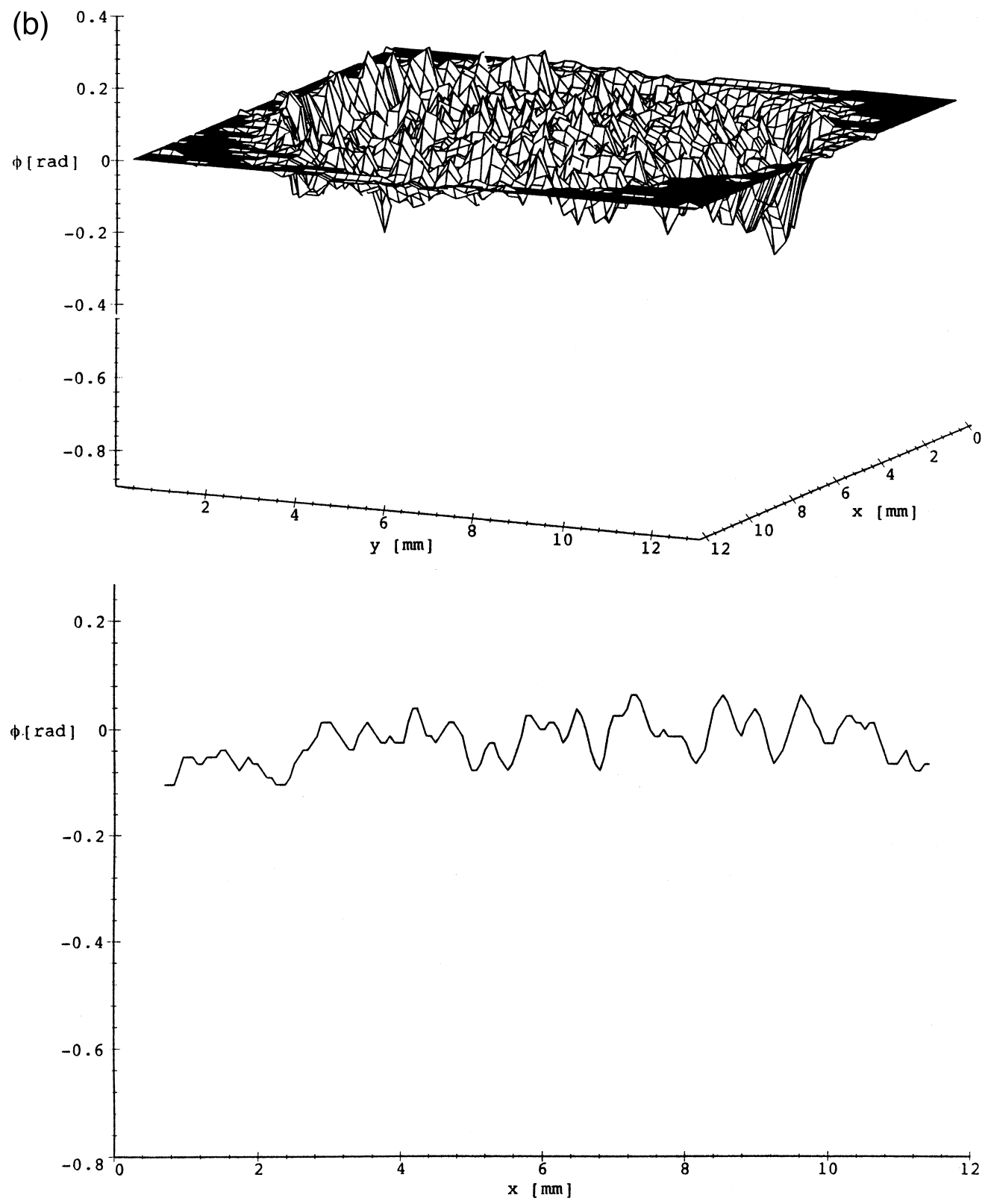

Fig. 4 (continued).

mounting is made of steatite, a material with low heat conductivity.

\subsection{Deformation of one spot}

In order to study the basic mechanism, the deformation of the mirror surface by means of a laser beam has been measured at first for a single spot. The beam diameter of the $\mathrm{Nd}$ :YAG ring oscillator was $250 \mu \mathrm{m}$; the powers used were 100 to $300 \mathrm{~mW}$. The irradiation lasted $15 \mathrm{~s}$ in order to obtain thermal equilibrium (see below). The measurements concern the central area of the mirror surface (limited by the camera aperture to about $0.8 \mathrm{~cm}^{2}$ ). 
In order to suppress the intensity noise of the CCD camera, 88 consecutive images of the phase front were averaged pixel by pixel. (For the picture format chosen there are just 88 memories available on the image processing card.) In addition, all the pixels with the same distance from the centre of the
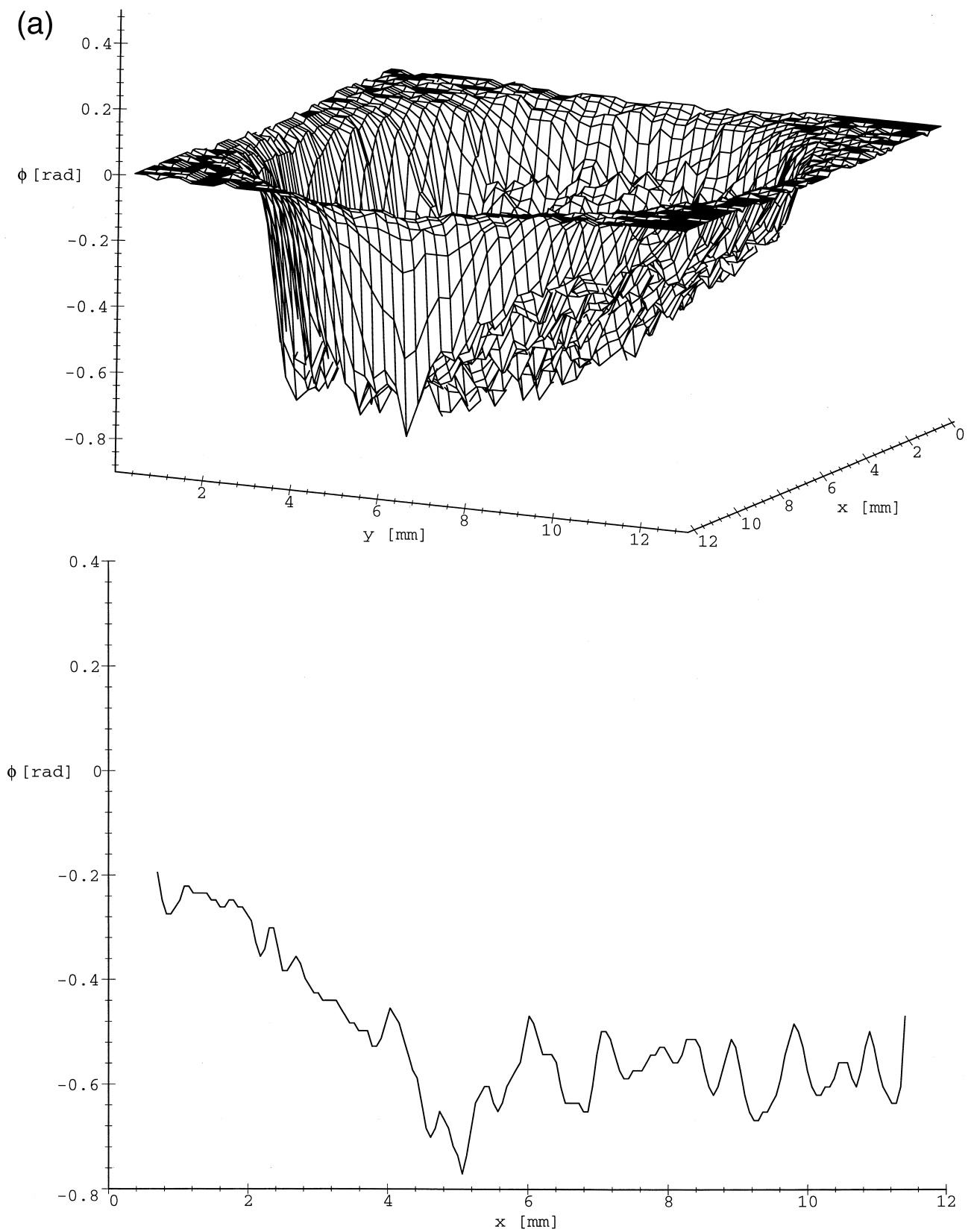

Fig. 5. Phase front of a tilted mirror; $\bar{\phi}=-0.527 \mathrm{rad}$ and $\sigma=0.504 \mathrm{rad} . x, y$, mirror co-ordinates; $\phi$, phase. (b) Corrected phase front of the tilted mirror (a); $\bar{\phi}=0.013 \mathrm{rad}$ and $\sigma=0.053 \mathrm{rad}$. 

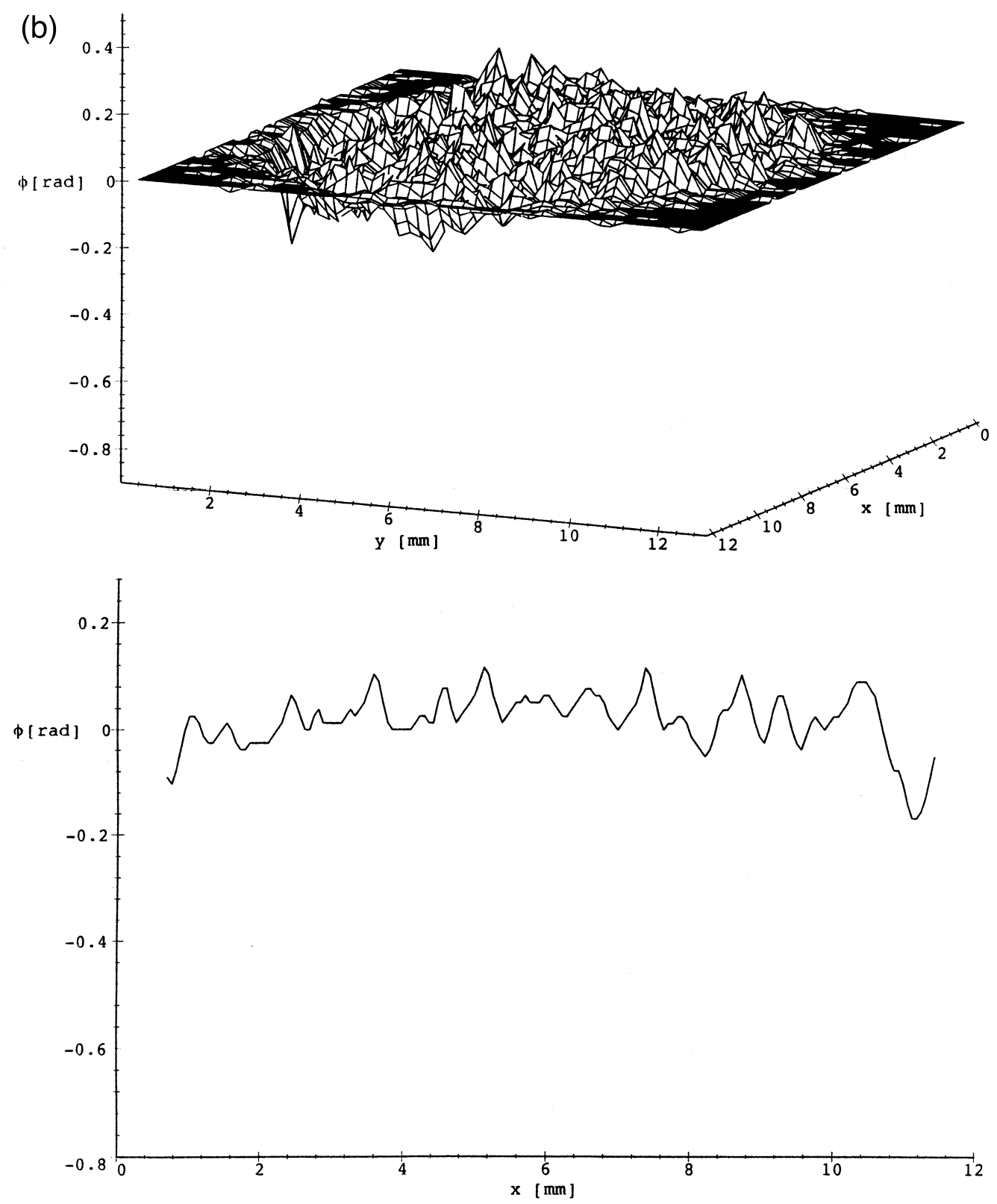

Fig. 5 (continued).

deformation were averaged because the problem is rotationally symmetric. The mirror expansion $\Delta z$ follows from the phase $\Delta \phi$ according to

$$
\Delta z=\frac{\Delta \phi}{4 \pi} \lambda
$$

In Fig. 2 the measurements for an irradiation power $P=100 \mathrm{~mW}$ are shown and compared with values calculated from the displacement equation of Hello and Vinet [10]. The arbitrary constant $C^{\prime}$ of their solution (equation 3.16 in [10]) was chosen as to obtain agreement in the wings of both curves. The 
discrepancy between calculated $(\Delta z=58 \mathrm{~nm})$ and observed $(\Delta z=45 \mathrm{~nm})$ maximum values originates in an assumption made to linearise the problem: The rise in temperature is assumed to be small compared to the environmental temperature ('low heating approximation'). This underestimates the heat losses by radiation, hence the theoretical values are too high. For $P=200$ and $300 \mathrm{~mW}$, the experimental values are $\Delta z=80$ and $130 \mathrm{~nm}$, respectively.

The transient behaviour of the deformation was observed, too. Here only a very small area around the deformation maximum was studied so that 1000 memories were available at the image processing card. This does not allow the averaging of several images, so the measured curves are much noisier. For each laser power an exposure time of some seconds (5 to $10 \mathrm{~s}$, according to the power) was sufficient to obtain saturation of deformation. Likewise the deformation recedes to the initial value within seconds after turning off the laser irradiation. The results are shown in Fig. 3.

\subsection{Correction of the whole phase front}

Next, the correction of the deformations of a complete phase front was studied. For that the control loop stabilising the interferometer to a dark fringe $(\bar{\phi}=0)$ was switched off thus allowing to shift the mirror as a whole. Then the adaptive control loop has to compensate not only for the phase front deformations but also to shift the complete front to the initial value.

For these experiments a cw Nd:YAG laser with $30 \mathrm{~W}$ output power was used in order to obtain the desired deformation as quickly as possible. The observed part of the surface was covered by a scan raster of 5 by 5 points. (This rather coarse grid results from the use of a $\mathrm{PC}$ with low computing power. Better PCs allow finer grids and thus much better control.) The area was scanned 50 times between two phase measurements. After each measurement of the phase, the exposure time $T$ was readjusted for each point according to the following algorithm:

$T_{n+1}=T_{n} \pm \frac{1}{2} \Delta T_{n+1} \quad(n=0,1,2)$

for $\Delta \phi<0$ and $\Delta \phi>0$, respectively, and $\Delta T_{n+1}$ $=\frac{1}{2} \Delta T_{n}$. The initial values $T_{0}$ and $\Delta T_{0}$ were both $8 \mathrm{~ms}$. Since only whole millisecond steps are al- lowed by the software a maximum of four corrections is possible. It turned out that this is sufficient.

Fig. 4 shows the correction of a parallel displaced mirror of poor surface quality. Before the correction (Fig. 4a) the mean value of the deformations was $\Delta z=-29.6$ (3.6) $\mathrm{nm}$ and the mean value of the large-scale surface roughness was $\sigma=28.0 \mathrm{~nm}$; afterwards (Fig. 4b) $\Delta z=-1.3 \quad$ (2.6) $\mathrm{nm}$ and $\sigma=2.8 \mathrm{~nm}$.

Fig. 5 shows the correction of a tilted mirror. Here $\Delta z=-26.5(5.5) \mathrm{nm}$ and $\sigma=25.4 \mathrm{~nm}$ before the correction (Fig. 5a), and $\Delta z=0.7(2.4) \mathrm{nm}$ and $\sigma=2.67 \mathrm{~nm}$ afterwards (Fig. 5b). As in Fig. 4, the remaining noise does not indicate short-scale deformations, but is due to intrinsic camera noise.

The extremely large fluctuations in laser power and mode behaviour made an optimal correction more difficult since the deformations responded very quickly to these fluctuations. But in both cases a convincing correction of the wavefront distortions was obtained after only four iterations.

\section{Conclusion and outlook}

In this study we have investigated a new method for the correction of wavefront distortions in laser interferometers. The correction is applied without contact by means of controlled thermal expansion of a mirror surface with a high power laser beam. First measurements show the feasibility of this 'thermally adaptive optics' method. Our results are in good agreement with the theory of thermoelastic deformations [10].

These experiments have been performed merely in order to prove the practicability of the method on principle. In order to be able to use this method in interferometric gravitational wave detectors several modifications and improvements are necessary. For instance, the mirrors are usually made of fused silica having a much lower thermal expansion than BK7. According to (1) approximately $400 \mathrm{~mW}$ absorbed power is required to produce a thermal deformation of $\lambda / 100$. Therefore $\mathrm{CO}_{2}$ or $\mathrm{CO}$ lasers are better suited than Nd:YAG lasers since their radiation is nearly completely absorbed by fused silica. Further- 
more lasers showing high stability in amplitude and mode behaviour are required for precise thermal deformation. This would allow the same exposure time to be used for each spot, producing the desired deformations by varying the laser power. For use in gravitational wave detectors the addition of a high resolution telescope is required in order to image the mirror surface to the distant wavefront sensor.

Advanced interferometers will face the problem that thermal lensing sets a power limit [8]. This problem has to be solved for the second generation of laser interferometric gravitational wave detectors in order to obtain the desired sensitivity. Our experiments show that it will be possible to develop and optimise a thermally adaptive system that can be used in these detectors.

\section{References}

[1] R.K. Tyson, Principles of Adaptive Optics, Academic Press, San Diego, 1991.

[2] G.C. Loos, Appl. Opt. 31 (1992) 6632.

[3] H. Lück, G. Heinzel, D. Maßß, K.-O. Müller, in: I. Ciufolini,
F. Fidecaro (Eds.), Gravitational Waves: Sources and Detectors, World Scientific, Singapore, 1997, 311.

[4] K. Danzmann, in: W.M. Folkner (Ed.), Laser Interferometer Space Antenna. AIP Conf. Proc. 456, AIP, Woodbury, NY, 1998, 3.

[5] J. Mizuno, Dissertation, Universität Hannover, 1995 and MPQ Report 203, 1995.

[6] C.M. Caves, Phys. Rev. D 23 (1981) 1693.

[7] G. Heinzel, K.A. Strain, J. Mizuno, K.D. Skeldon, B. Willke, W. Winkler, R. Schilling, A. Rüdiger, K. Danzmann, Phys. Rev. Lett. 81 (1998) 5493.

[8] K.A. Strain, K. Danzmann, J. Mizuno, P.G. Nelson, A. Rüdiger, R. Schilling, W. Winkler, Phys. Lett. A 194 (1994) 124.

[9] W. Winkler, K. Danzmann, A. Rüdiger, R. Schilling, Phys. Rev. A 44 (1991) 7022.

[10] P. Hello, J.-Y. Vinet, J. Physique 51 (1990) 2243.

[11] K. Danzmann, in: E. Coccia, G. Pizzella, F. Ronga (Eds.), Gravitational Wave Experiments, World Scientific, Singapore, 1995, 100.

[12] I. Freitag, A. Tünnermann, H. Welling, Opt. Commun. 115 (1995) 511

[13] O.S. Brozeck, M. Peterseim, K. Danzmann, B. Willlke, I. Freitag, S. Knoke, in: E. Coccia, G. Pizzella, G. Veneziano (Eds.), Gravitational Waves, World Scientific, Singapore, 1998, 360.

[14] S. Rowan, S.M. Twyford, J. Hough, in: E. Coccia, G. Pizzella, G. Veneziano (Eds.), Gravitational Waves, World Scientific, Singapore, 1998, 363. 